\title{
Prevalence and Time Course of Post-Stroke Pain: A Multicenter Prospective Hospital-Based Study
}

\author{
Stefano Paolucci, MD, ${ }^{*}$ Marco losa, PhD, ${ }^{*}$ \\ Danilo Toni, MD, ${ }^{\dagger}$ Piero Barbanti, MD, ${ }^{\ddagger}$ \\ Paolo Bovi, MD, § Anna Cavallini, MD, "ृ E. \\ Candeloro," Alessia Mancini, MD, ${ }^{\dagger}$ Mauro \\ Mancuso, MD, " Serena Monaco, MD, \\ Pieroni, MD, ${ }^{\dagger}$ Serena Recchia, MD, ${ }^{* \star}$ Maria Sessa, \\ MD, ${ }^{\dagger+}$ Davide Strambo, MD, ${ }^{\dagger+}$ Michele Tinazzi, \\ MD, ${ }^{* *}$ Giorgio Cruccu, MD, ${ }^{\dagger}$ and Andrea Truini, MD, \\ $\mathrm{PhD}^{\dagger}$ on behalf of the Neuropathic pain special \\ interest group of the Italian Neurological Society
}

\section{*Fondazione Santa Lucia IRCCS, Rome, Italy; \\ ${ }^{\dagger}$ Department of Neurology and Psychiatry, Sapienza University, Rome, Italy; ${ }^{\ddagger}$ IRCCS San Raffaele Pisana, Rome, Italy; §Ospedale Civile Maggiore Borgo Trento, Verona, Italy; " Casimiro Mondino, IRCCS Istituto Neurologico Nazionale, Pavia, Italy; "CRT-Rehabilitation Center of Terranuova Bracciolini, Arezzo, Italy; "IIOspedale Civico, Palermo, Italy; ${ }^{\star \star}$ Department of Neurological and Movement Sciences, Anatomy and Histology Section, University of Verona, Verona, Italy; \\ ${ }^{+\dagger}$ IRCCS Ospedale San Raffaele, Milano, Italy}

Correspondence to: Andrea Truini, Department of Neurology and Psychiatry, Sapienza University, Viale Università 30, 00185, Rome, Italy. Tel: +39-06-49914438; Fax: +39-06-49914525; E-mail: andrea.truini@uniroma1.it.

Conflicts of interest: There are no conflicts of interest to report.

\section{Abstract \\ Objective. Pain prevalence data for patients at vari- ous stages after stroke.}

Design. Repeated cross-sectional, observational epidemiological study.

Setting. Hospital-based multicenter study.

Subjects. Four hundred forty-three prospectively enrolled stroke survivors.

Methods. All patients underwent bedside clinical examination. The different types of post-stroke pain (central post-stroke pain, musculoskeletal pains, shoulder pain, spasticity-related pain, and headache) were diagnosed with widely accepted criteria during the acute, subacute, and chronic stroke stages. Differences among the three stages were analyzed with $\chi^{2}$-tests.

Results. The mean overall prevalence of pain was $29.56 \%(14.06 \%$ in the acute, $42.73 \%$ in the subacute, and $31.90 \%$ in the chronic post-stroke stage). Time course differed significantly according to the various pain types $(P<0.001)$. The prevalence of musculoskeletal and shoulder pain was higher in the subacute and chronic than in the acute stages after stroke; the prevalence of spasticity-related pain peaked in the chronic stage. Conversely, headache manifested in the acute post-stroke stage. The prevalence of central post-stroke pain was higher in the subacute and chronic than in the acute poststroke stage. Fewer than $25 \%$ of the patients with central post-stroke pain received drug treatment.

Conclusions. Pain after stroke is more frequent in the subacute and chronic phase than in the acute phase, but it is still largely undertreated.

Key Words. Stroke; Central Post-Stroke Pain; Hemiplegic Shoulder Pain; Spasticity

Introduction

Pain is a common event in stroke survivors. Post-stroke pain manifests in various ways including neuropathic pain, namely central post-stroke pain (CPSP), nociceptive pains (musculoskeletal pains, shoulder pain, spasticityrelated pain) and headache [1]. The estimated prevalence rates range from $10 \%$ to $45.8 \%$ for post-stroke pain and from $1 \%$ to $12 \%$ for CPSP [2,3]. This variability reflects the different methodological approaches. Most studies are based on retrospective data, and neglected to use clinical examination or widely agreed criteria for distinguishing the different types of pain, or the recently proposed criteria for diagnosing neuropathic pain $[1,4,5]$.

Although clinicians commonly believe that headache has an early onset whereas some post-stroke pains (including CPSP and spasticity-related pain) have a delayed onset, no study has directly investigated the 


\section{Paolucci et al.}

time course of the various post-stroke pains. Having more information on these events might improve the clinical management of this complication that adversely affects stroke patients' quality of life.

This prospective, observational, repeated crosssectional, hospital-based, multicenter study was designed to seek more reliable information on the prevalence and time course of the various post-stroke pains. All patients enrolled underwent bedside clinical examination, and using widely accepted criteria we diagnosed the different types of pain during the acute, subacute, and chronic stages after stroke.

\section{Methods}

All patients consecutively admitted to the stroke units and stroke rehabilitation divisions (both in the ward and in the outpatient service) at eight Italian hospitals between January 2010 and July 2012 were considered eligible for the study (Figure 1). The study was conducted in accordance with the Helsinki Declaration, and informed consent was obtained before the examination. The study was approved by the Institutional Review Boards at the participating hospitals.

Inclusion criteria were a stroke diagnosis (defined as a sudden, non-convulsive, focal neurological deficit

\section{Acute units}

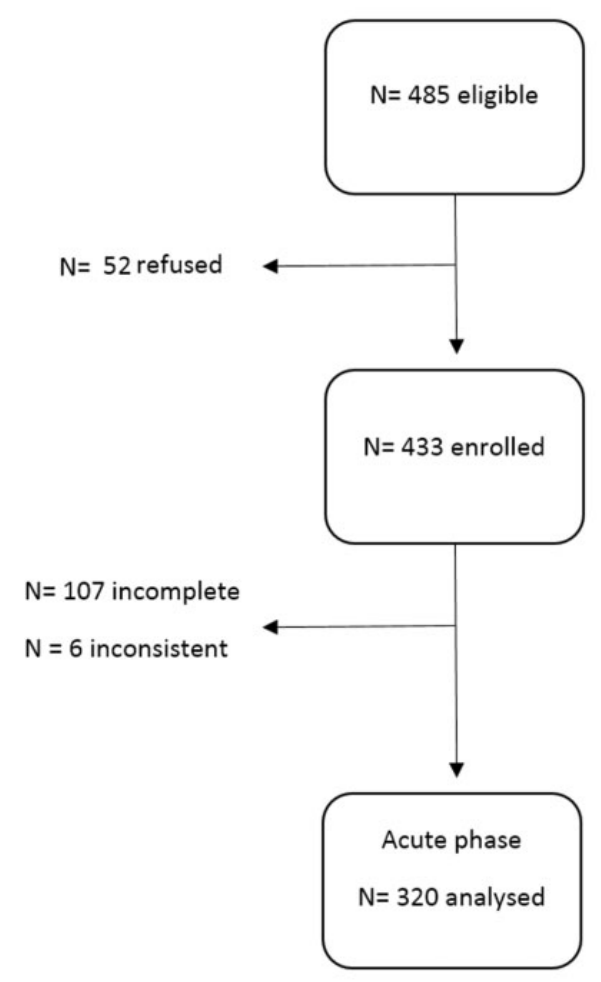

persisting for $>24$ hours [6]), age over 18 years, and patient's consent to a free participation to the study. The diagnosis of stroke was based on clinical history, clinical examination, and neuroradiological findings. Patients were distinguished according to onset as having acute (within 14 days after the event), subacute (from day 15 to day 90), or chronic stroke (more than 90 days after the event). Exclusion criteria were as follows: negative neuroimaging findings, previous cerebrovascular accidents, subarachnoid hemorrhage, presence of coma or language disorder with severe comprehension deficit (Token test, cut-off score of 29), history of severe depression before stroke (needing prolonged antidepressant treatment and/or admission to a psychiatric ward), other chronic severe disabling diseases (including severe Parkinson's disease, polyneuropathy, severe cardiac, liver, or renal failure, cancer, and limb amputation). We also excluded patients who complained of chronic pain before the stroke, defined as any persistent or recurrent pain experienced within the past 3 months and needing prolonged/ chronic analgesic medication. Antithrombotic stroke therapy was not considered a reason to exclude patients.

After enrolment all patients underwent a detailed neurological examination, using bedside tools and focused assessment of sensory disturbances. Touch was

\section{Rehabilitation units}

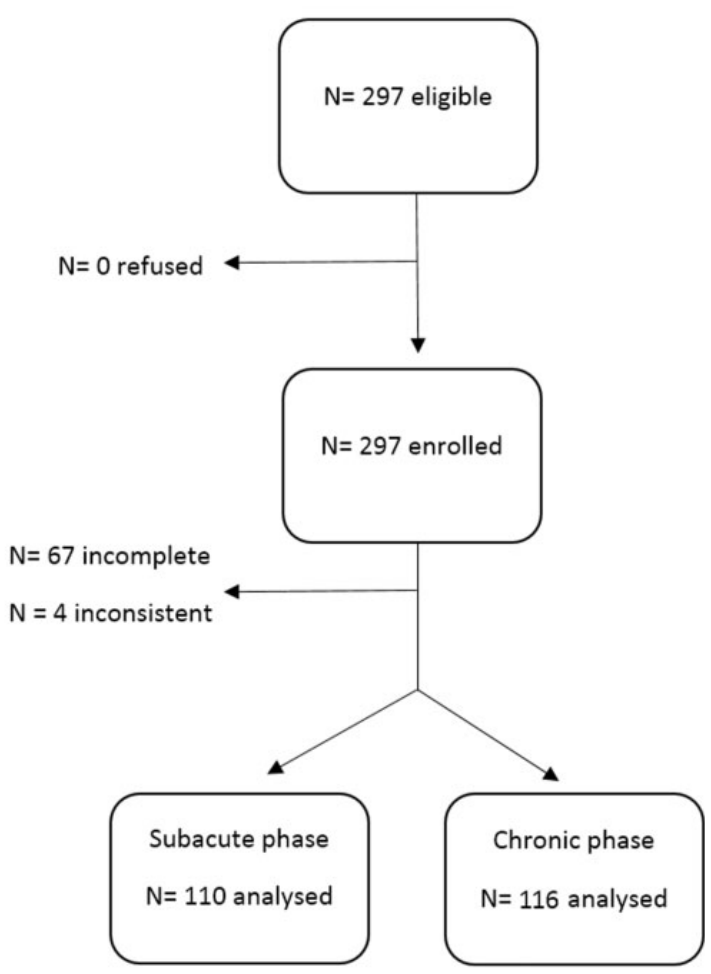

Figure 1 Study flow-chart. 


\section{Time Course of Post-Stroke Pain}

investigated with a piece of cotton wool, vibration with a tuning fork $(128 \mathrm{~Hz})$, pinprick sensation with a wooden cocktail stick, and thermal sensation with test tubes containing warm and cold water. Patients were examined for negative (tactile, pinprick, and thermal hypoesthesia), and positive symptoms (constant pain, paroxysmal pain, allodynia, and pinprick hyperalgesia). The presence and severity of negative symptoms were assessed by comparing the affected side with the mirror image on the normal side. Using a structured interview we collected demographic data and information on the type and side of stroke, onset interval, severity of neurological impairment (with the National Institutes of Health Stroke Scale [NIHSS]) and disability (with the Barthel Index), comorbidities and concomitant treatment, and pain at the time of examination.

CPSP was diagnosed according to the grading system for the diagnosis of neuropathic pain $[1,4,5]$. Patients were diagnosed as suffering from definite neuropathic pain when examination excluded other likely causes of pain, pain had a plausible neuroanatomical distribution, a diagnosis of stroke, a plausible neuroanatomical distribution confirmed by clinical findings, a DN4 questionnaire score of $\geq 4[7,8]$, and a compatible vascular lesion on imaging. To assess the different symptoms and severity of neuropathic pain we used the Italian version of the Neuropathic Pain Symptom Inventory (NPSI), a self-administered questionnaire consisting of 12 items related to the different symptoms. Ten items assess the intensity of pain by a $0-10$ numerical scale $(0=$ no sensation, $10=$ worst possible pain) and two items assess the frequency of pain $[9,10]$. Each NPSI subscore was calculated.

We diagnosed nociceptive pains by clinical history and examination and distinguished musculoskeletal pains (including low back pain and joint pains), shoulder pain, and spasticity-related pain. Musculoskeletal and shoulder pains were diagnosed using combined interview, physical examination, and diagnostic tests as appropriate [11]. We diagnosed spasticity-related pain when pain involved spasticity-affected limbs (spasticity was defined as $\geq 1$ at any one of the major joints in the arms and legs, using the 0-5 range on the Modified Ashworth Scale). The diagnosis of stroke-related headache adhered to the International Headache Classification (headache attributed to ischemic stroke and headache attributed to non-traumatic intracranial hemorrhage) [12].

\section{Statistical Analysis}

Parametric and non-parametric tests were used to compare demographic, clinical, and functional data for the three subgroups (acute, subacute, and chronic stroke stages). Binary data related to the presence of each type of pain were reported as percentages and analyzed with Pearson $\chi^{2}$-test applied to a $3 \times 2$ cross table using the stroke stage (acute, subacute, or chronic) as the three-variant factor and the presence or not of that type of pain as the binary factor. Pearson $\chi^{2}$-test applied to $2 \times 2$ cross tables was then used for post hoc analysis applying Bonferroni correction on the alpha-level. Pearson $\chi^{2}$-test was also applied in the frequency analysis of NPSI items.

Forward binary linear regression was performed to identify the dichotomized factors associated to overall pain or subtypes of pain. The investigated risk factors were: age (<65 years or not), gender, type of stroke (hemorrhagic vs ischemic), and severity of stroke (as assessed with $\mathrm{NIHSS}$ in the acute stage and Barthel Index in subacute and chronic stages). Odds ratio was computed as the exponential of beta-coefficient of regressions. The 95\% confidence interval (C195\%) was computed as well.

\section{Results}

During the 2-year study, 485 eligible patients were identified in stroke units and neurological departments and 297 patients in rehabilitation units. Among the eligible patients, 52 refused to be enrolled, 174 patients had incomplete data, and 10 inconsistent data (Figure 1). The data for 546 assessments were analyzed (these data concerned 443 patients, 103 of whom were assessed more than one time during the acute and subacute or chronic stages). Demographic and clinical data are reported in Table 1.

The mean prevalence of the various pain types studied was $29.59 \%$, but the percentage ranged widely in the three stroke stages: $14.06 \%$ in the acute, $42.73 \%$ in the subacute, and $31.90 \%$ in the chronic stage. Pain was significantly associated with the stroke stage $(P<0.001$, Figure 2). Whereas prevalence of musculoskeletal pains, shoulder pain, spasticity-related pain, and headache differed significantly across the three stroke stages $(P<0.05$, Figure 2), CPSP prevalence, though higher in the subacute and chronic stages than in the acute stage, did not significantly differ across the three stages $(P=0.1)$.

The frequencies of the different types of pain, as assessed with NPSI, did not significantly differ (they ranged from $35 \%$ to $67 \%$ ). All the different types of pain had a median value of severity between 5 and 6 (except pain evoked by cold stimuli), with a score ranging between 2 and 7 (Table 2).

Post hoc analyses showed that pain overall, as well as musculoskeletal pains and shoulder pain, had a higher prevalence in the subacute and chronic stages than in the acute stage $(P<0.001)$. Conversely, the prevalence of spasticity-related pain was higher in the chronic than in other stages $(P<0.001)$. Headache tended to decrease without significant post hoc comparisons. In 97 out of 546 assessments (17.8\%), patients complained of more than one type of pain.

The time course of the different types of pain in the 103 patients assessed more than once was similar to that of 


\section{Paolucci et al.}

Table 1 Demographical and clinical features of patients (mean \pm standard deviation are reported for age and time from onset)

\begin{tabular}{llll} 
FeaturelPhase & Acute phase & Subacute phase & Chronic phase \\
\hline $\mathrm{N}^{\circ}$ assessments & 320 & 110 & 116 \\
Age (years) & $67.16 \pm 14.08$ & $67.60 \pm 14.18$ & $66.59 \pm 14.73$ \\
Time from stroke (days) & $2.10 \pm 2.83$ & $47.77 \pm 24.42$ & $174.89 \pm 107.71$ \\
Gender & $61.6 \%$ men & $59.1 \%$ men & $58.6 \%$ men \\
Type of stroke & $92.8 \%$ ischemic & $90.0 \%$ ischemic & $87.9 \%$ ischemic \\
Side of hemiparesis/hemiplegia & $53.1 \%$ right & $61.8 \%$ right & $49.6 \%$ right \\
& $46.9 \%$ left & $38.2 \%$ left & $50.4 \%$ left \\
\hline
\end{tabular}

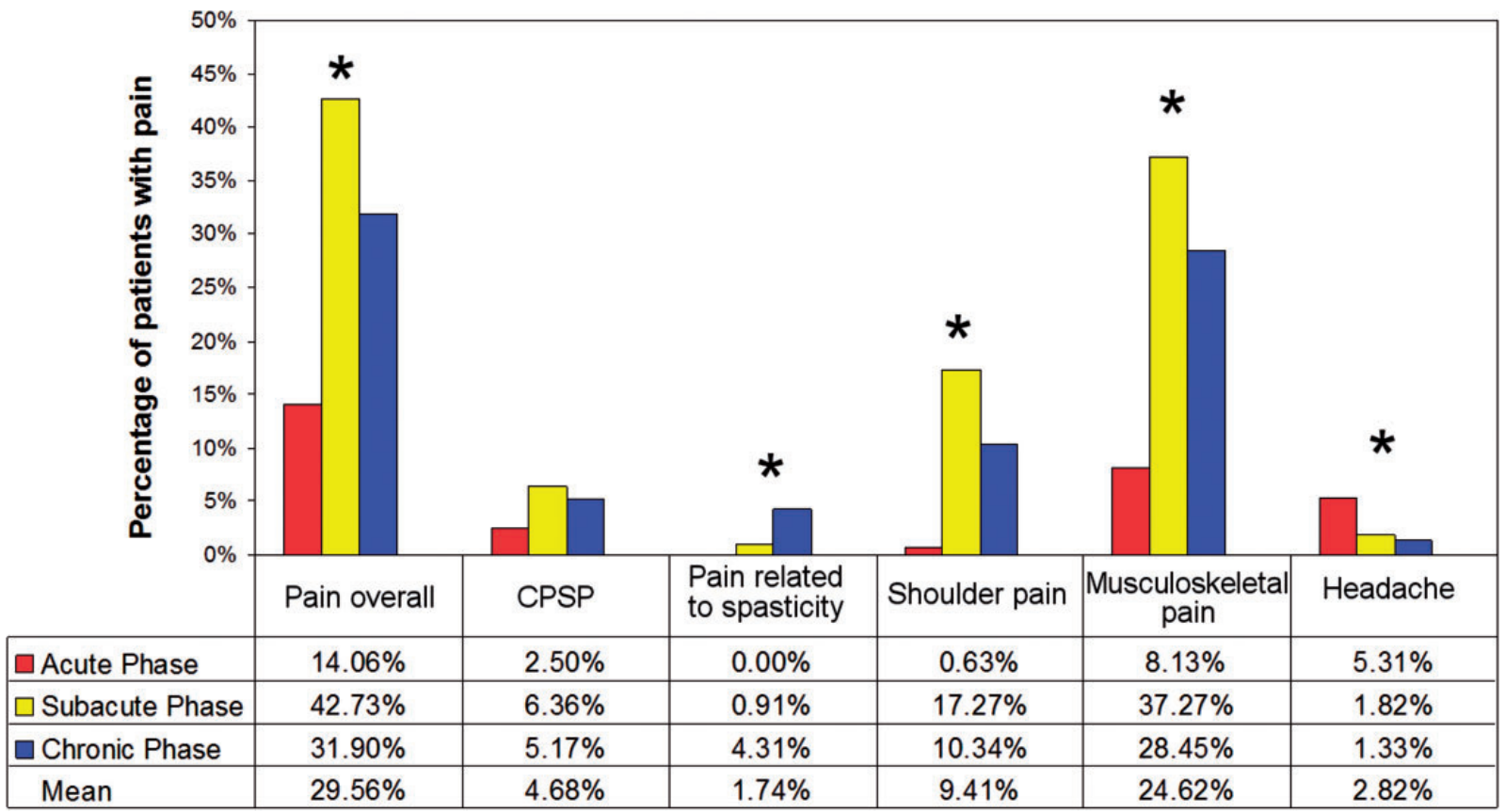

Figure 2 Prevalence of post-stroke pain and the different pain types. Asterisks indicate a statistically significant difference among the different stroke stages $(P<0.05$, by $\chi 2$-test). CPSP $=$ central post-stroke pain.

the entire sample, except the spasticity-related pain (Figure S1, Supplementary Data file). The frequency of this type of pain was higher in the chronic stage than in the other stroke stages; this difference nevertheless failed to reach the statistical significance.

Table 3 reports the risk factors associated with overall pain and the specific types of pain. Young patients ( $<65$ years) and women had a probability 1.7 times higher to suffer from pain. Patients with hemorrhagic stroke and women had a higher risk of suffering from headache $(P<0.05)$. Similar results were found in the subgroup of patients in the acute stage $(P<0.05)$. In the subacute stage, none of the investigated factors were significantly associated with pain. In the chronic stage we found a mild association between age lower than 65 years and CPSP. We did not find any association between pain and the severity of stroke, as assessed with NIHSS in the acute stage, and Barthel Index in the subacute and chronic stage.

In the acute stage only one patient with CPSP underwent analgesic treatment with acetaminophen. In the subacute and chronic phases, four of the 13 patients with CPSP (30.8\%) were treated: two patients with pregabalin, one with gabapentin plus oxcarbazepine, and one with non-steroidal anti-inflammatory drugs.

\section{Discussion}

Our repeated cross-sectional, hospital-based, multicenter study, using bedside clinical examination and widely agreed criteria for diagnosing post-stroke pains, provides reliable information confirming in a large 


\section{Time Course of Post-Stroke Pain}

population that pain is a common complication after stroke. In the prospectively enrolled patients hospitalized or attending Italian stroke units and stroke rehabilitation divisions, it affected about $30 \%$ of stroke survivors, and the prevalence peaked in the subacute stroke stage (40\% of patients).

The mean 30\% prevalence of post-stroke pain in our population is in line with several studies reporting a prevalence ranging from $10 \%$ to $45.8 \%$ [1-3,13]. With respect to previous studies mainly focused on one type for pain investigated in a specific phase of stroke, our study provides a new insight detailing a clear time course for the different pain types, showing that the prevalence of post-stroke pain differs strikingly across the acute, subacute, and chronic stroke stages.

Table 2 NPSI findings

\begin{tabular}{|c|c|c|}
\hline NPSI descriptors & Patients* $(\%)$ & $\begin{array}{l}\text { Severity }{ }^{\dagger} \\
\text { (median [first; } \\
\text { third quartile]) }\end{array}$ \\
\hline Q1: Burning & 49.2 & $6(4 ; 7)$ \\
\hline Q2: Squeezing & 44.3 & $5(3 ; 7)$ \\
\hline Q3: Pressure & 46.4 & $5(3 ; 7)$ \\
\hline Q5: Electric shocks & 49.2 & $5(3 ; 7)$ \\
\hline Q6: Stabbing & 35.1 & $5(2 ; 7)$ \\
\hline Q8: Evoked by brushing & 39.7 & $5(3 ; 7)$ \\
\hline Q9: Evoked by pressure & 44.8 & $5(2 ; 7)$ \\
\hline Q10: Evoked by cold stimuli & 30.1 & $4(2 ; 6)$ \\
\hline Q11: Pins and needles & 55.9 & $5(3 ; 7)$ \\
\hline Q12: Tingling & 66.8 & $6(3 ; 7)$ \\
\hline
\end{tabular}

Q4 and Q7 not reported. They refer to the temporal sequence of spontaneous ongoing pain (Q1/Q2/Q3) and paroxysmal pain (Q5/Q6).

${ }^{*}$ Percentage of patients who reported a score $>0$.

${ }^{\dagger}$ Median (first; third quartiles) values of patients who reported a score $>0$.
The CPSP mean prevalence we found in our hospitalbased study is lower than that reported in previous epidemiological studies $[3,13]$. The contrasting results presumably reflect the different methodological approaches. Whereas we explicitly diagnosed CPSP with clinical examination and validated questionnaires, previous studies frequently assessed pain and neuropathic pain by mail questionnaire alone. The DN4 questionnaire that we used in our study was specifically designed to diagnose neuropathic pain, thus increasing diagnostic specificity and reducing false-positive diagnoses, an especially important advantage in a condition producing different types of pain, such as stroke. The DN4 is a valid tool for screening patients with suspected neuropathic pain and achieves high diagnostic specificity and sensitivity (about 80\%) [7]. In our study the CPSP prevalence ranged between $2.5 \%$ and $6.4 \%$ across the three post-stroke stages. Although the few subjects with CPSP reduced test power, and thus prevented a statistically significant difference in prevalence across the three stroke stages, we identified a trend in which CPSP prevalence seems to be higher in the subacute and chronic stages than in the acute stage. The fact that CPSP is more frequent in the subacute and chronic stages is in line with previous studies showing that neuropathic pain, probably owing to plastic changes in the somatosensory and pain pathways, is a delayed complication after stroke [14,15]. Our study provides the previously unreported finding of NPSI analysis in patients with CPSP. The frequency and the severity of the different types of pain did not differ. However, given that only a few patients suffered from CPSP, caution is needed in interpreting these results. Further studies, collecting a large sample of patients with CPSP, might reach more reliable conclusions on CPSP.

When we investigated the various nociceptive post-stroke pains, we found that the most frequent pain type was musculoskeletal pain (low back pain, joint pains). Its prevalence reached about $37 \%$ between the first and second month after stroke, and then decreased to $28 \%$. This time course probably reflects the observation that

Table 3 Risk factors for the different types of pain

\begin{tabular}{lllll} 
Phase & Type of pain & Factor & $P$ & OR (Cl95\%) \\
\hline All patients & Overall pain & Female gender & 0.007 & $1.735(1.159-2.599)$ \\
& & Age $<65$ & 0.009 & $1.723(1.159-2.588)$ \\
& Headache & Female gender & 0.006 & $3.998(1.498-10.671)$ \\
Acute & Overall pain & Hemorrhagic stroke & 0.049 & $3.217(1.003-10.314)$ \\
& & Female gender & 0.006 & $2.518(1.304-4.862)$ \\
& Headache & Hemorrhagic stroke & 0.035 & $3.027(1.082-8.469)$ \\
Chronic & CPSP & Female gender & 0.015 & $3.774(1.293-11.012)$ \\
& & Hemorrhagic stroke & 0.036 & $4.500(1.099-18.421)$ \\
& & Age $<65$ & 0.040 & $9.848(1.105-87.781)$
\end{tabular}

Data obtained with forward binary logistic regression ( $P$ value refers to the last value, OR is the odds ratio obtained as exponential of coefficient beta of regression, $\mathrm{Cl} 95 \%$ is the $95 \%$ confidence interval). No significant risk factor was identified in the subacute phase. 


\section{Paolucci et al.}

musculoskeletal disorders receive more adequate attention in rehabilitation wards than in stroke wards [16].

Our findings show that the prevalence of shoulder pain is negligible in the acute stage, then markedly increases in the subacute stage. In this later stage we found a prevalence of $18 \%$, similar to that reported in a recent large population-based study in patients at 1 month after stroke $[17,18]$. The time course we found confirmed shoulder pain as a typically delayed complication after stroke. Although this time course might merely reflect rotator cuff disorders, adhesive capsulitis, shoulder subluxation and shoulder muscle spasticity, it indirectly implies that this type of pain might depend partly on central sensitization [19]. Accordingly, in our study the time course of shoulder pain paralleled that for CPSP.

The prevalence of spasticity-related pain was higher in the chronic than in the acute post-stroke stage. The pathophysiological mechanism underlying this type of pain is potentially multifactorial and strictly related to how stroke units, rehabilitation centers, and caregivers at home manage secondary complications [20,21], including musculoskeletal consequences related to abnormal postures (soft tissue and joint stiffness; joint subluxations; and several lower-limb sequelae, such as equinovarus, hyperextended knee, and adducted hip) and secondary tissue damage from abnormal postures, such as decubitus ulcers. The increasing prevalence of pain related to spasticity in the chronic stroke stage could therefore reflect poor attention to correct positioning after discharge from the rehabilitation hospital and poor compliance with specific drug treatment.

Our finding that headache complicates mainly the acute stroke stage, agrees with previous studies [22]. The prevalence rate we found is lower than that reported in a previous large study including both hemorrhagic and ischemic stroke (5\% vs 18\%) [23]. The contrasting results might depend on the few patients with hemorrhagic stroke we enrolled. Accordingly, the headache prevalence we found matches that from a previous study collecting only patients with ischemic stroke [24].

Unexpectedly, only $30 \%$ of patients with CPSP in the acute stroke stage and $11 \%$ in the subacute and chronic stages underwent analgesic treatment. The reasons why patients with post-stroke pain receive inadequate therapy could be the difficulty in diagnosing the different pain types related to stroke, the insufficient awareness about CPSP, and the poor therapeutic options [25].

When we analyzed the risk factors, we found that age and gender influenced post-stroke pain. Patients younger than 65 years and women have a higher risk of suffering from pain. Although these findings partly argue against earlier observation, they are in line with a recent study reporting a significant association between gender, age, and post-stroke pain [26]. We have also found that patients with hemorrhagic stroke had a higher risk of headache. This association is probably due to local distention, distortion, deformation, or stretching of pain- sensitive intracranial structures [27]. In our study women had an higher risk of suffering from stroke-associated headache. This finding probably suggests that besides the well-known higher incidence of migraine and tension type headache in women, gender might also influence symptomatic headaches.

Our study showing that the prevalence of stroke-related pain is higher in the subacute and chronic stages than in the acute stage and that each type of pain follows a distinct time course should help neurologists managing this common post-stroke complication so that their patients benefit from a better quality of life.

\section{Supplementary Data}

Supplementary Data are available online at http:// www.painmedicine.oxfordjournals.org.

\section{References}

1 Klit H, Finnerup NB, Jensen TS. Central post-stroke pain: Clinical characteristics, pathophysiology, and management. Lancet Neurol 2009;8:857-68.

2 Kalita J, Kumar B, Misra UK, Pradhan PK. Central post stroke pain: Clinical, MRI, and SPECT correlation. Pain Med 2011;12(2):282-8.

3 Hansen AP, Marcussen NS, Klit H, et al. Pain following stroke: A prospective study. Eur J Pain 2012; 16:1128-36.

4 Treede RD, Jensen TS, Campbell JN, et al. Neuropathic pain: Redefinition and a grading system for clinical and research purposes. Neurology 2008; 70:1630-5.

5 Vaegter HB, Andersen PG, Madsen MF, Handberg G, Enggaard TP. Prevalence of neuropathic pain according to the IASP grading system in patients with chronic non-malignant pain. Pain Med 2014;15:120-7.

6 Foulkes MA, Wolf PA, Price TR, Mohr JP, Hier DB. The stroke data bank: Design, methods, and baseline characteristics. Stroke 1988;19:547-54.

7 Bouhassira D, Attal N, Alchaar H, et al. Comparison of pain syndromes associated with nervous or somatic lesions and development of a new neuropathic pain diagnostic questionnaire (DN4). Pain 2005;114:29-36.

8 Markman JD, Kress BT, Frazer M, et al. Screening for neuropathic characteristics in failed back surgery syndromes: Challenges for guiding treatment. Pain Med 2015;16:520-30.

9 Bouhassira D, Attal N, Fermanian J, et al. Development and validation of the Neuropathic Pain Symptom Inventory. Pain 2004;108:248-57. 


\section{Time Course of Post-Stroke Pain}

10 Padua L, Briani C, Jann S, et al. Validation of the Italian version of the Neuropathic Pain Symptom Inventory in peripheral nervous system diseases. Neurol Sci 2009;30:99-106.

11 Viana R, Pereira S, Mehta S, Miller T, Teasell R. Evidence for therapeutic interventions for hemiplegic shoulder pain during the chronic stage of stroke: A review. Top Stroke Rehabil 2012;19:514-22.

12 The International Classification of Headache Disorders, 3rd edition (beta version). Cephalalgia 2013;33:629-808.

13 Klit $H$, Finnerup NB, Andersen G, Jensen TS. Central poststroke pain: A population-based study. Pain 2011;152:818-24.

14 Hansson P. Post-stroke pain case study: Clinical characteristics, therapeutic options and long-term follow-up. Eur J Neurol 2004;11(suppl 1):22-30.

15 Truini A, Garcia-Larrea L, Cruccu G. Reappraising neuropathic pain in humans-how symptoms help disclose mechanisms. Nat Rev Neurol 2013;9:572-82.

16 McGregor AH, Probyn K, Cro S, et al. Rehabilitation following surgery for lumbar spinal stenosis. Cochrane Database Syst Rev 2013;12:CD009644.

17 Lindgren I, Jonsson AC, Norrving B, Lindgren A. Shoulder pain after stroke: A prospective population-based study. Stroke 2007;38:343-8.

18 Ratnasabapathy Y, Broad J, Baskett J, et al. Shoulder pain in people with a stroke: A populationbased study. Clin Rehabil 2003;17:304-11.
19 Roosink M, Renzenbrink GJ, Buitenweg JR, et al. Somatosensory symptoms and signs and conditioned pain modulation in chronic post-stroke shoulder pain. J Pain 2011;12:476-85.

20 Sheean DG. Is spasticity painful? Eur J Neurol 2009;16:157-8.

21 Ward AB. A literature review of the pathophysiology and onset of post-stroke spasticity. Eur $J$ Neurol 2012;19:21-7.

22 Goddeau RP, Alhazzani A. Headache in stroke: A review. Headache 2013;53:1019-22.

23 Kumral E, Bogousslavsky J, Van MG, Regli F, Pierre P. Headache at stroke onset: The Lausanne Stroke Registry. J Neurol Neurosurg Psychiatry 1995; 58:490-2.

24 Chen PK, Chiu PY, Tsai IJ, et al. Onset headache predicts good outcome in patients with first-ever ischemic stroke. Stroke 2013;44:1852-8.

25 Backonja MM, Serra J. Pharmacologic management part 2: Lesser-studied neuropathic pain diseases. Pain Med 2004;5(suppl 1):S48-59.

26 O'Donnell MJ, Diener HC, Sacco RL, et al. PRoFESS Investigators. Chronic pain syndromes after ischemic stroke: PRoFESS trial. Stroke 2013; 44:1238-43.

27 Leira R, Castellanos M, Alvarez-Sabín J, et al. Headache in cerebral hemorrhage is associated with inflammatory markers and higher residual cavity. Headache 2005;45:1236-43. 